\title{
Comunicação
}

\section{Inquérito sobre prevalência do tabagismo na classe médica brasileira}

\author{
A.P. MIRRA, J . ROSEMBERG \\ Comissão de Combate ao Tabagismo da Associação Médica Brasileira
}

\begin{abstract}
RESUMO - ОвJ etIvos. Apresentar os resultados do inquérito sobre a prevalência atual de fumantes entre os médicos do Brasil, segundo suas especialidades, realizado no período de maio a outubro de 1996.

MÉtodo. A amostra utilizada foi de respostas espontâneas, constituída por 11.909 médicos associados pagantes da Associação Médica Brasileira, das várias Unidades da Federação e especialidades médicas.

Resultados. Constatou-se que 759 (6,4\%) são fumantes regulares e $11.150(93,6 \%)$ são não-fumantes; 4.085 médicos (34,3\%) são ex-fumantes. Os maiores índices de não-fumantes estão entre os associados de sociedades de especialidades que possuem programas efetivos de controle do tabagismo (pneumologia, cancerologia, car-
\end{abstract}

\section{NTRODUÇÃO}

O tabagismo é considerado a maior causa isolada evitável de doença e morte, porquanto são atribuídas a esse vício $90 \%$ dos casos de câncer de pulmão, $86 \%$ de bronquite crônica e enfisema, 25\% dos processos isquêmicos do coração e $30 \%$ dos cânceres extrapulmonares. No mundo, ocorrem 3 milhões de óbitos ( $5 \%$ da mortalidade geral) e, no Brasil, 80 a 100 mil mortes (10,9\% da mortalidade geral), anualmente. Em nosso país, 30 milhões de indivíduos com mais de 15 anos de idade são fumantes (32,6\% da população), sendo $40,4 \%$ muIheres e dois terços moradores de zonas urbanas.

Sendo o tabagismo uma pandemia e, portanto, um problema de saúde pública, deve ser combatido energicamente. Os médicos têm papel muito importante nos programas antifumo ${ }^{1,2}$, devendo ser mobilizados para uma atuação, principalmente, preventiva, com ações educativas junto à população, de modo a influir na diminui ção do número de pessoas que se iniciam no tabagismo; além disso, podem agir, individualmente, junto aos fumantes para que abandonem o cigarro.

O médico tem notável poder de persuasão, ajudando o tabagista a vencer a ni cotino-dependência, pois as pessoas, em geral, esperam receber auxílio diologia e otorrinolaringologia). As regiões geográficas do Norte, Nordeste e Centro-Oeste apresentaram as maiores prevalências de fumantes. O início do fumar está mais presente no grupo de $10-19$ anos $(72,6 \%)$, com distribuição semelhante entre os sexos. Nenhum médico do sexo feminino iniciou o seu vício de fumar antes dos $\mathbf{1 0}$ anos de idade.

Conclusão. É de grande importância os médicos receberem informações sobre tabagismo, bem como a realização de programas de seu controle nas sociedades de especialidades e nas federadas da Associação Médica Brasileira.

UnITermos: Tabagismo. Prevalência. Classe médica brasileira.

desse profissional para seus problemas de saúde, levando o paciente à convicção de que, abandonando o fumo, ele viverá melhor e mais tempo.

O aconselhamento médico é eficaz, desde que exercido de maneira constante. Em vários países, estudos mostraram que se cada médico se dedicar, durante dois a três minutos, a cada paciente, levando informações, consegue uma redução de $10 \%$ no número de fumantes entre seus doentes. Daí a importância de sua participação nos programas naci onais de combate à epi demia tabágica, e o êxito desse enfrentamento se deve a uma classe médica constituída por não-fumantes.

O médico que fuma destrói toda a credibilidade da mensagem de que fumar é lesivo à saúde e apressa a morte, desacreditando a luta antitabágica. Programas informativos sobre tabagismo devem ser introduzidos nas escolas médicas, em suas atividades curriculares, o que facilitará, de muito, a formação de uma população médica livre do tabaco. A Organização Mundial de Saúde (OMS), entre suas várias recomendações, advoga que os médicos devem dar o exemplo, abstendo-se de fumar.

$\mathrm{Na}$ maioria dos países desenvolvidos, a proporção de médicos fumantes, que era alta, diminuiu sensivelmente, após a tomada de conhecimento dos vários relatórios sobre os malefícios do taba- 
gismo, divulgados pel as organizações médicas e de saúde internacionais. No Brasil, o índice de médicos tabagistas variou de $20 \%$ a $50 \%{ }^{3-9}$, segundo inquéritos realizados por amostragem, na maioria das vezes, utilizando eventos científicos, como congressos médi cos realizados nos anos de 1970 a 1991. Para especialistas pneumologistas, o índice de fumantes variou de $6,4 \%$ a $17 \%(1991 / 95)^{10,11}$.

O objetivo deste trabalho é apresentar os resultados do inquérito realizado sobre a prevalência atual de fumantes entre os médicos no Brasil, segundo suas especialidades.

\section{MATERIAL E MÉTODOS}

O inquérito sobre a prevalência do tabagismo na classe médica brasileira teve o patrocínio da Associação Médica Brasileira (AMB), por meio de sua Comissão de Combate ao Tabagismo, suas F ederadas e Sociedades de Especialidades, colaboração da Fundação Oncocentro de São Paulo e apoi o da Sanofi Winthrop Farmacêutica Ltda.

A população-alvo definida é constituída por médicos associados pagantes da AMB, filiados nas várias Unidades da F ederação (anexos 1 e 2) e especialidades médicas (anexo 3), totalizando 51.558 médicos.

A amostra utilizada foi de respostas espontâneas, constituída por 11.909 médicos (23,1\% da populaçãoalvo), distribuídos pelas Unidades da Federação (anexos 1 e 2) e especialidades médicas (anexo 3).

Para fins de análise, considerou-se uma estratificação posterior. Cada médico constante da população-alvo recebeu, pelo correio (mala direta), um formulário padrão (anexo 4), com oito itens para serem respondidos, com envelope-resposta selado.

As variáveis constantes do formulário são: nome do médico, idade, sexo, especialidade, condição de fumante regular e de ex-fumante, idade em que iniciou fumar e local onde exerce sua atividade médica (cidade e U nidade da F ederação).

Para a análise estatística, foi utilizado teste de associação, usando a estatística qui-quadrado $\left(\chi^{2}\right)$, eliminando os dados ignorados.

O período de realização do inquérito foi de maio a outubro de 1996. As respostas espontâneas dos médicos, em número de 11.909, se apresentaram, segundo o sexo: masculino, 9.105 (76,5\%), e feminino, 2.804 (23,5\%). A distribuição, segundo o grupo etário e sexo, é dada pela tabela 1 .

\section{RESULTADOS}

Na amostra, constataram-se 759 (6,4\%) fumantes regulares e 11.150 (93,6\%) não-fumantes; 4.085 médicos (34,3\%) foram fumantes (ex-fumantes).

\begin{tabular}{|c|c|c|c|}
\hline \multirow[b]{2}{*}{$\begin{array}{l}\text { Grupo etário } \\
\text { (anos) }\end{array}$} & \multicolumn{2}{|c|}{ Sexo } & \multirow[b]{2}{*}{$\begin{array}{c}\text { Total } \\
\text { № }(\%)\end{array}$} \\
\hline & $\begin{array}{l}\text { Masculino } \\
\text { № (\%) }\end{array}$ & $\begin{array}{c}\text { Feminino } \\
\text { № }(\%)\end{array}$ & \\
\hline $\begin{array}{l}<35 \\
35-69 \\
>69 \\
\text { Ignorada }\end{array}$ & $\begin{aligned} 780 & (8,6) \\
7.640 & (83,9) \\
670 & (7,3) \\
15 & (0,2)\end{aligned}$ & $\begin{aligned} 660 & (23,5) \\
2.110 & (75,2) \\
27 & (1,0) \\
7 & (0,3)\end{aligned}$ & $\begin{array}{r}1.440(12,1) \\
9.750(81,9) \\
697(5,8) \\
22(0,2)\end{array}$ \\
\hline Total & $9.105(100)$ & $2.804(100)$ & $11.909(100)$ \\
\hline
\end{tabular}

A distribuição dos médicos fumantes atuais, segundo o sexo e o grupo etário, é dada pela tabela 2 .

Os médicos fumantes atuais, segundo a especialidade médica, constam na tabela 3 .

A distribuição dos médicos fumantes atuais, segundo a Unidade da Federação, é dada pela tabela 4.

A distribuição dos médicos fumantes atuais, segundo a área geográfica do país, é dada pela tabela 5 .

A distribuição dos fumantes atuais, segundo o grupo etário do início do fumar, é dada pela tabela 6.

Os médicos não-fumantes, segundo o sexo e o grupo etário, constam na tabela 7 .

A distribuição dos médicos não-fumantes, segundo a especialidade médica, é dada pela tabela 8 .

A distribui ção dos médicos não-fumantes, segundo a Unidade da Federação, é dada pela tabela 9.

Os médicos não-fumantes, segundo a área geográfica do país, constam na tabela 10.

A distribuição dos médicos ex-fumantes, segundo o sexo e o grupo etário, é dada pela tabela 11.

A distribuição dos médicos ex-fumantes, segundo a especialidade médica, é dada pela tabela 12 .

Os médicos ex-fumantes, segundo a Unidade da F ederação, constam na tabela 13.

A distribuição dos médicos ex-fumantes, segundo a área geográfica do país, é dada pela tabela 14.

A distribuição dos médicos ex-fumantes, segundo o sexo e o grupo etário do início de fumar, é dada pela tabela 15 .

A distribuição dos médicos fumantes e ex-fumantes, segundo o sexo e o grupo etário do início de fumar, é dada pela tabela 16.

\section{DISCUSSÃO E CONCLUSÃO}

Os países que apresentam programas de controle de tabagismo bem estruturados, e quando a participação dos médicos é efetiva, conseguiram uma diminuição de fumantes entre esses profissionais. Nos EUA e na Inglaterra, a prevalência do 


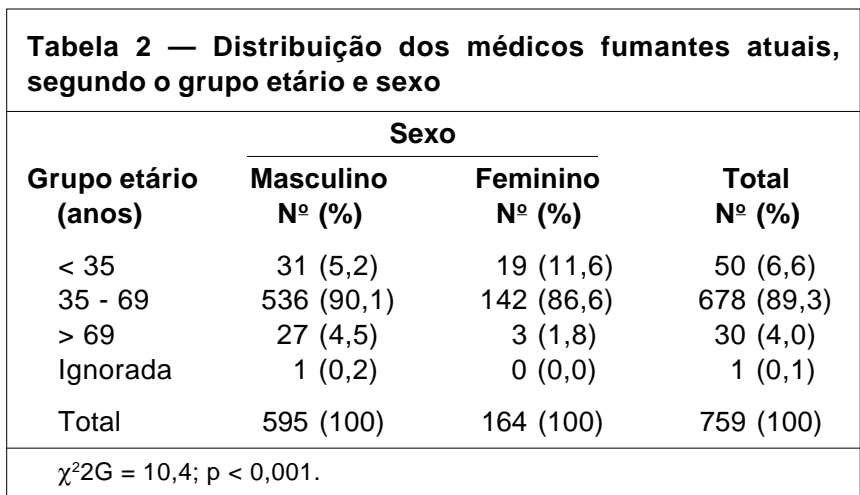

Tabela 3 - Distribuição dos médicos fumantes atuais, segundo a especialidade médica

\begin{tabular}{|c|c|c|c|}
\hline Especialidade & № & № de respostas & $\%$ \\
\hline Administração em Saúde & 7 & 56 & 12,5 \\
\hline Alergologia / Imunopatologia & 2 & 51 & 3,9 \\
\hline Anestesiologia & 39 & 453 & 8,6 \\
\hline Cancerologia & 4 & 109 & 3,7 \\
\hline Cardiologia & 29 & 682 & 4,3 \\
\hline Cirurgia Cabeça e Pescoço & 2 & 28 & 7,1 \\
\hline Cirurgia Cardiovascular & 1 & 31 & 3,2 \\
\hline Cirurgia Digestiva & 3 & 77 & 3,9 \\
\hline Cirurgia Geral & 51 & 569 & 9,0 \\
\hline Cirurgia Neurológica & 7 & 125 & 5,6 \\
\hline Cirurgia Pediátrica & 6 & 62 & 9,7 \\
\hline Cirurgia Plástica & 19 & 201 & 9,5 \\
\hline Cirurgia Vascular / Angiologia & 11 & 169 & 6,5 \\
\hline Clínica Médica & 97 & 1.285 & 7,5 \\
\hline Dermatologia & 25 & 375 & 6,7 \\
\hline Endocrinologia / Metabologia & 11 & 209 & 5,3 \\
\hline Endoscopia & 3 & 38 & 7,9 \\
\hline Gastroenterologia & 26 & 344 & 7,6 \\
\hline Genética Clínica & 1 & 7 & 14,3 \\
\hline Geriatria & 2 & 81 & 2,5 \\
\hline Ginecologia / Obstetrícia & 101 & 1.642 & 6,2 \\
\hline Hematologia / Hemoterapia & 2 & 77 & 2,6 \\
\hline Homeopatia & 3 & 95 & 3,2 \\
\hline Infectologia & 3 & 58 & 5,2 \\
\hline Mastologia & 0 & 24 & 00 \\
\hline Medicina de Tráfego & 0 & 2 & 00 \\
\hline Medicina Desportiva & 0 & 3 & 00 \\
\hline Medicina do Trabalho & 12 & 133 & 9,0 \\
\hline Medicina Intensiva / UTI & 1 & 14 & 7,1 \\
\hline Medicina Legal & 1 & 9 & 11,1 \\
\hline Nefrologia & 5 & 136 & 3,7 \\
\hline Neurologia/ Neurofisiologia & 21 & 202 & 10,4 \\
\hline Nutrologia & 0 & 5 & 00 \\
\hline Oftalmologia & 20 & 537 & 3,7 \\
\hline \multicolumn{4}{|l|}{ Ortopedia/Traumatologia/ } \\
\hline Reumatologia & 52 & 576 & 9,0 \\
\hline Otorrinolaringologia & 14 & 320 & 4,3 \\
\hline Patologia / Citopatologia & 10 & 130 & 7,7 \\
\hline Patologia Clínica & 12 & 125 & 9,6 \\
\hline Pediatria & 61 & 1.425 & 4,3 \\
\hline Pneumologia / Tisiologia & 6 & 273 & 2,2 \\
\hline Psiquiatria & 48 & 484 & 9,9 \\
\hline Radiologia & 21 & 341 & 6,2 \\
\hline Urologia & 12 & 219 & 5,5 \\
\hline Especialidade ignorada & 8 & 127 & 6,3 \\
\hline Total & 759 & 11.909 & 6,4 \\
\hline
\end{tabular}

Tabela 4 - Distribuição dos médicos fumantes atuais segundo a Unidade da Federação

\begin{tabular}{lrrr} 
Unidade da Federação & № & № de respostas & $\%$ \\
Acre & 1 & 5 & 20,0 \\
Alagoas & 3 & 57 & 5,3 \\
Amapá & 0 & 3 & 0,0 \\
Amazonas & 2 & 47 & 4,3 \\
Bahia & 21 & 423 & 5,0 \\
Ceará & 5 & 100 & 5,0 \\
Distrito Federal & 17 & 233 & 7,3 \\
Espírito Santo & 10 & 239 & 4,2 \\
Goiás & 9 & 228 & 3,9 \\
Maranhão & 1 & 12 & 8,3 \\
Mato Grosso & 5 & 46 & 10,9 \\
Mato Grosso do Sul & 19 & 227 & 8,4 \\
Minas Gerais & 76 & 1.187 & 6,4 \\
Pará & 5 & 60 & 8,3 \\
Paraíba & 9 & 150 & 6,0 \\
Paraná & 58 & 900 & 6,4 \\
Pernambuco & 20 & 257 & 7,8 \\
Piauí & 9 & 110 & 8,2 \\
Rio de Janeiro & 37 & 564 & 6,6 \\
Rio Grande do Norte & 15 & 206 & 7,3 \\
Rio Grande do Sul & 95 & 1.674 & 5,7 \\
Rondônia & 4 & 44 & 9,1 \\
Roraima & 2 & 5 & 40,0 \\
Santa Catarina & 26 & 614 & 4,2 \\
São Paulo & 303 & 4.362 & 6,9 \\
Sergipe & 7 & 145 & 4,8 \\
Tocantins & 0 & 11 & 0,0 \\
Total & 759 & 11.909 & 6,4 \\
\hline
\end{tabular}

Tabela 5 - Distribuição dos médicos fumantes atuais, segundo a região geográfica do país

\begin{tabular}{|lrcc|}
\hline Região geográfica & № & № de respostas & $\%$ \\
Norte & 14 & 175 & 8,0 \\
Nordeste & 90 & 1.460 & 6,2 \\
Centro-Oeste & 50 & 734 & 6,8 \\
Sudeste & 426 & 6.352 & 6,7 \\
Sul & 179 & 3.188 & 5,6 \\
Total & 759 & 11.909 & 6,4 \\
\hline
\end{tabular}

Tabela 6 - Distribuição dos médicos fumantes atuais, segundo o grupo etário do início de fumar

\begin{tabular}{|c|c|c|c|}
\hline \multirow[b]{2}{*}{$\begin{array}{l}\text { Grupo etário } \\
\text { (anos) }\end{array}$} & \multicolumn{2}{|c|}{ Sexo } & \multirow[b]{2}{*}{$\begin{array}{l}\text { Total } \\
\text { № (\%) }\end{array}$} \\
\hline & $\begin{array}{c}\text { Masculino } \\
\text { № (\%) }\end{array}$ & $\begin{array}{c}\text { Feminino } \\
\text { № (\%) }\end{array}$ & \\
\hline$<10$ & $4(0,7)$ & -- & $4(0,5)$ \\
\hline $10-19$ & $439(73,8)$ & $112(68,3)$ & $551(72,6)$ \\
\hline $20-29$ & $124(20,8)$ & $45(27,4)$ & $169(22,3)$ \\
\hline$>29$ & $15(2,5)$ & $4(2,4)$ & $19(2,5)$ \\
\hline Ignorado & $13(2,2)$ & $3(1,8)$ & $16(2,1)$ \\
\hline Total & $595(100)$ & $164(100)$ & 759 (100) \\
\hline
\end{tabular}




\begin{tabular}{|c|c|c|c|}
\hline \multicolumn{4}{|c|}{$\begin{array}{l}\text { Tabela } 7-\text { Distribuição dos } \\
\text { segundo o sexo e o grupo etário }\end{array}$} \\
\hline \multirow[b]{2}{*}{$\begin{array}{l}\text { Grupo etário } \\
\text { (anos) }\end{array}$} & \multicolumn{2}{|c|}{ Sexo } & \multirow[b]{2}{*}{$\begin{array}{l}\text { Total } \\
\text { № (\%) }\end{array}$} \\
\hline & $\begin{array}{c}\text { Masculino } \\
\text { № (\%) }\end{array}$ & $\begin{array}{c}\text { Feminino } \\
\text { № }(\%)\end{array}$ & \\
\hline$<35$ & $749(8,8)$ & $641(24,3)$ & $1.390(12,5)$ \\
\hline $35-69$ & $7.104(83,5)$ & $1.968(74,5)$ & $9.072(81,3)$ \\
\hline$>69$ & $643(7,5)$ & $24(0,9)$ & $667(6,0)$ \\
\hline Ignorado & $14(0,2)$ & $7(0,3)$ & $21(0,2)$ \\
\hline Total & $8.510(100)$ & $2.640(100)$ & $11.150(100)$ \\
\hline
\end{tabular}

\begin{tabular}{|c|c|c|c|}
\hline Especialidade & № & № de respostas & $\%$ \\
\hline Administração em Saúde & 49 & 56 & 87,5 \\
\hline Alergologia/Imunopatologia & 49 & 51 & 96,1 \\
\hline Anestesiologia & 414 & 453 & 91,4 \\
\hline Cancerologia & 105 & 109 & 96,3 \\
\hline Cardiologia & 653 & 682 & 95,7 \\
\hline Cirurgia Cabeça e Pescoço & 26 & 28 & 92,9 \\
\hline Cirurgia Cardiovascular & 30 & 31 & 96,8 \\
\hline Cirurgia Digestiva & 74 & 77 & 96,1 \\
\hline Cirurgia Geral & 518 & 569 & 91,0 \\
\hline Cirurgia Neurológica & 118 & 125 & 94,4 \\
\hline Cirurgia Pediátrica & 56 & 62 & 90,3 \\
\hline Cirurgia Plástica & 182 & 201 & 90,5 \\
\hline Cirurgia Vascular / Angiologia & 158 & 169 & 93,5 \\
\hline Clínica Médica & 1.188 & 1.285 & 92,5 \\
\hline Dermatologia & 350 & 375 & 93,3 \\
\hline Endocrinologia / Metabologia & 198 & 209 & 94,7 \\
\hline Endoscopia & 35 & 38 & 92,1 \\
\hline Gastroenterologia & 318 & 344 & 92,4 \\
\hline Genética Clínica & 6 & 7 & 85,7 \\
\hline Geriatria & 79 & 81 & 97,5 \\
\hline Ginecologia / Obstetrícia & 1.541 & 1.642 & 93,8 \\
\hline Hematologia / Hemoterapia & 75 & 77 & 97,4 \\
\hline Homeopatia & 92 & 95 & 96,8 \\
\hline Infectologia & 55 & 58 & 94,8 \\
\hline Mastologia & 24 & 24 & 100,0 \\
\hline Medicina de Tráfego & 2 & 2 & 100,0 \\
\hline Medicina Desportiva & 3 & 3 & 100,0 \\
\hline Medicina do Trabalho & 121 & 133 & 91,0 \\
\hline Medicina Intensiva / UTI & 13 & 14 & 92,9 \\
\hline Medicina Legal & 8 & 9 & 88,9 \\
\hline Nefrologia & 131 & 136 & 96,3 \\
\hline Neurologia / Neurofisiologia & 181 & 202 & 89,6 \\
\hline Nutrologia & 5 & 5 & 100,0 \\
\hline Oftalmologia & 517 & 537 & 96,3 \\
\hline Ortopedia/Traumatologia / & & & \\
\hline Reumatologia & 524 & 576 & 91,0 \\
\hline Otorrinolaringologia & 306 & 320 & 95,7 \\
\hline Patologia / Citopatologia & 120 & 130 & 92,3 \\
\hline Patologia Clínica & 113 & 125 & 90,4 \\
\hline Pediatria & 1.364 & 1.425 & 95,7 \\
\hline Pneumologia / Tisiologia & 267 & 273 & 97,8 \\
\hline Psiquiatria & 436 & 484 & 90,1 \\
\hline Radiologia & 320 & 341 & 93,8 \\
\hline Urologia & 207 & 219 & 94,5 \\
\hline Especialidade ignorada & 119 & 127 & 93,7 \\
\hline Total & 11.150 & 11.909 & 93,6 \\
\hline
\end{tabular}

Tabela 9 - Distribuição dos médicos não-fumantes, segundo a Unidade da Federação

\begin{tabular}{|lrrr|}
\hline Unidade da Federação & \multicolumn{1}{c}{ № } & № de respostas & $\%$ \\
Acre & 4 & 5 & 80,0 \\
Alagoas & 54 & 57 & 94,7 \\
Amapá & 3 & 3 & 100,0 \\
Amazonas & 45 & 47 & 95,7 \\
Bahia & 402 & 423 & 95,0 \\
Ceará & 95 & 100 & 95,0 \\
Distrito Federal & 216 & 233 & 92,7 \\
Espírito Santo & 229 & 239 & 95,8 \\
Goiás & 219 & 228 & 96,1 \\
Maranhão & 11 & 12 & 91,7 \\
Mato Grosso & 41 & 46 & 89,1 \\
Mato Grosso do Sul & 208 & 227 & 91,6 \\
Minas Gerais & 1.111 & 1.187 & 93,6 \\
Pará & 55 & 60 & 91,7 \\
Paraíba & 141 & 150 & 94,0 \\
Paraná & 842 & 900 & 93,6 \\
Pernambuco & 237 & 257 & 92,2 \\
Piauí & 101 & 110 & 91,8 \\
Rio de Janeiro & 527 & 564 & 93,4 \\
Rio Grande do Norte & 191 & 206 & 92,7 \\
Rio Grande do Sul & 1.579 & 1.674 & 94,3 \\
Rondônia & 40 & 44 & 90,9 \\
Roraima & 3 & 5 & 60,0 \\
Santa Catarina & 588 & 614 & 95,8 \\
São Paulo & 4.059 & 4.362 & 93,1 \\
Sergipe & 138 & 145 & 95,2 \\
Tocantins & 11 & 11 & 100,0 \\
Total & 11.150 & 11.909 & 93,6 \\
\hline
\end{tabular}

Tabela 10 - Distribuição dos médicos não-fumantes, segundo a região geográfica do país

\begin{tabular}{|lrcc|}
\hline Região geográfica & \multicolumn{1}{c}{ № } & № de respostas & $\%$ \\
Norte & 161 & 175 & 92,0 \\
Nordeste & 1.370 & 1.460 & 93,8 \\
Centro-Oeste & 684 & 734 & 93,2 \\
Sudeste & 5.926 & 6.352 & 93,3 \\
Sul & 3.009 & 3.188 & 94,4 \\
Total & 11.150 & 11.909 & 93,6 \\
\hline
\end{tabular}

fumar entre os médicos é baixa $(9 \%)^{12}$. No Brasil, poucos inquéritos foram realizados e os índices de tabagistas variaram de $20 \%$ a $50 \%$, no período de 1970 a $1991^{3-9}$. As amostras utilizadas tiveram métodos diversos e as populações-alvo foram, na maioria das vezes, aquelas presentes em congressos médicos.

E sta pesquisa utilizou uma população-al vo mais ampla e definida, constituída pelos médicos associados pagantes da AMB, distribuídos nas várias Unidades da Federação e especialidades médicas. A amostra obtida foi de respostas espontâneas, de 11.909 médicos (23,1\% da população-alvo), resultado esse considerado satisfatório. Apesar de este inquérito ter usado técnica de amostragem, do tipo 


\begin{tabular}{|lccc|}
\hline \multicolumn{2}{|c|}{$\begin{array}{l}\text { Tabela } 11-\text { Distribuição dos médicos } \\
\text { segundo o sexo e o grupo etário }\end{array}$} & ex-fumantes, \\
\hline \multicolumn{4}{|c|}{ Sexo } \\
Grupo etário & $\begin{array}{c}\text { Masculino } \\
\text { (anos) }\end{array}$ & Fominino & Total \\
$<35$ & $95(2,6)$ & $55(10,8)$ & $150(3,7)$ \\
$35-69$ & $3.132(87,6)$ & $448(87,8)$ & $3.580(87,6)$ \\
$>69$ & $342(9,6)$ & $6(1,2)$ & $348(8,5)$ \\
Ignorado & $6(0,2)$ & $1(0,2)$ & $7(0,2)$ \\
Total & $3.575(100)$ & $510(100)$ & $4.085(100)$ \\
\hline$\chi^{2} 2 G=117,19 ; p<0,0001$. & \\
\hline
\end{tabular}

Tabela 12-Distribuição dos médicos ex-fumantes, segundo a especialidade médica

\begin{tabular}{lrrr|}
\hline Especialidade & № & № de respostas & $\%$ \\
Administração em Saúde & 14 & 56 & 25,0 \\
Alergologia/Imunopatologia & 19 & 51 & 37,3 \\
Anestesiologia & 149 & 453 & 32,9 \\
Cancerologia & 35 & 109 & 32,1 \\
Cardiologia & 235 & 682 & 34,5 \\
Cirurgia Cabeça e Pescoço & 6 & 28 & 21,4 \\
Cirurgia Cardiovascular & 9 & 31 & 29,0 \\
Cirurgia Digestiva & 34 & 77 & 44,2 \\
Cirurgia Geral & 218 & 569 & 38,3 \\
Cirurgia Neurológica & 42 & 125 & 33,6 \\
Cirurgia Pediátrica & 19 & 62 & 38,6 \\
Cirurgia Plástica & 63 & 201 & 31,3 \\
Cirurgia Vascular / Angiologia & 68 & 169 & 40,2 \\
Clínica Médica & 483 & 1.285 & 37,6 \\
Dermatologia & 89 & 375 & 23,7 \\
Endocrinologia / Metabologia & 60 & 209 & 28,7 \\
Endoscopia & 10 & 38 & 26,3 \\
Gastroenterologia & 123 & 344 & 35,8 \\
Genética Clínica & 3 & 7 & 42,9 \\
Geriatria & 27 & 81 & 33,3 \\
Ginecologia / Obstetrícia & 581 & 1.642 & 35,4 \\
Hematologia / Hemoterapia & 21 & 77 & 27,3 \\
Homeopatia & 30 & 95 & 31,6 \\
Infectologia & 12 & 58 & 20,7 \\
Mastologia & 10 & 24 & 41,7 \\
Medicina de Tráfego & 2 & 2 & 100,0 \\
Medicina Desportiva & 1 & 3 & 33,3 \\
Medicina do Trabalho & 48 & 133 & 36,1 \\
Medicina Intensiva / UTI & 5 & 14 & 35,7 \\
Medicina Legal & 2 & 9 & 22,2 \\
Nefrologia & 50 & 136 & 36,8 \\
Neurologia / Neurofisiologia & 59 & 202 & 29,2 \\
Nutrologia & 3 & 5 & 60,0 \\
Oftalmologia & 187 & 537 & 34,8 \\
Ortopedia/Traumatologia/ & & & \\
Reumatologia & 209 & 576 & 36,3 \\
Otorrinolaringologia & 101 & 320 & 31,6 \\
Patologia / Citopatologia & 38 & 130 & 29,2 \\
Patologia Clínica & 46 & 125 & 36,8 \\
Pediatria & & 1.425 & 27,6 \\
Pneumologia / Tisiologia & 273 & 36,3 \\
Psiquiatria & 484 & 39,5 \\
Radiologia & 341 & 42,8 \\
Urologia & & 11.909 & 41,6 \\
Especialidade ignorada & & 41,7 \\
Total & & & 34,3 \\
\hline
\end{tabular}

Tabela 13 - Distribuição dos médicos ex-fumantes, segundo a Unidade da Federação

\begin{tabular}{|lrrc|}
\hline Unidade da Federação & \multicolumn{1}{c}{ № } & № de respostas & $\%$ \\
Acre & 3 & 5 & 60,0 \\
Alagoas & 17 & 57 & 29,8 \\
Amapá & 2 & 3 & 66,7 \\
Amazonas & 19 & 47 & 40,4 \\
Bahia & 150 & 423 & 35,5 \\
Ceará & 35 & 100 & 35,0 \\
Distrito Federal & 74 & 233 & 31,8 \\
Espírito Santo & 75 & 239 & 31,4 \\
Goiás & 76 & 228 & 33,3 \\
Maranhão & 5 & 12 & 41,7 \\
Mato Grosso & 17 & 46 & 37,0 \\
Mato Grosso do Sul & 66 & 227 & 29,1 \\
Minas Gerais & 392 & 1.187 & 33,0 \\
Pará & 18 & 60 & 30,0 \\
Paraíba & 42 & 150 & 28,0 \\
Paraná & 285 & 900 & 31,7 \\
Pernambuco & 106 & 257 & 41,2 \\
Piauí & 48 & 110 & 43,6 \\
Rio de Janeiro & 216 & 564 & 38,3 \\
Rio Grande do Norte & 62 & 206 & 30,1 \\
Rio Grande do Sul & 573 & 1.674 & 34,2 \\
Rondônia & 10 & 44 & 22,7 \\
Roraima & 1 & 5 & 20,0 \\
Santa Catarina & 221 & 614 & 36,0 \\
São Paulo & 1.524 & 4.362 & 34,9 \\
Sergipe & 42 & 145 & 29,0 \\
Tocantins & 6 & 11 & 54,5 \\
Total & 4.085 & 11.909 & 34,3 \\
\hline
\end{tabular}

Tabela 14 - Distribuição dos médicos ex-fumantes, segundo a região geográfica do país

\begin{tabular}{|lrcc|}
\hline Região geográfica & \multicolumn{1}{c}{ № } & № de respostas & $\%$ \\
Norte & 59 & 175 & 33,7 \\
Nordeste & 507 & 1.460 & 34,7 \\
Centro-Oeste & 233 & 734 & 31,7 \\
Sudeste & 2.207 & 6.352 & 34,7 \\
Sul & 1.079 & 3.188 & 33,8 \\
Total & 4.085 & 11.909 & 34,3 \\
\hline
\end{tabular}

\begin{tabular}{|lccc|}
\hline \multicolumn{4}{|c|}{$\begin{array}{l}\text { Tabela 15-Distribuição dos médicos ex-fumantes, segundo } \\
\text { o sexo e o grupo etário do início de fumar }\end{array}$} \\
\hline \multicolumn{4}{|c}{ Sexo } \\
$\begin{array}{c}\text { Grupo etário } \\
\text { (anos) }\end{array}$ & $\begin{array}{c}\text { Masculino } \\
\text { № (\%) }\end{array}$ & $\begin{array}{c}\text { Feminino } \\
\text { № }(\%)\end{array}$ & $\begin{array}{c}\text { Total } \\
\text { № }(\%)\end{array}$ \\
$<10$ & $7(0,2)$ & --- & $7(0,2)$ \\
$10-19$ & $2.664(74,5)$ & $336(65,9)$ & $3.000(73,4)$ \\
$20-29$ & $569(15,9)$ & $134(26,3)$ & $703(17,2)$ \\
$>29$ & $57(1,6)$ & $11(2,2)$ & $68(1,7)$ \\
Ignorado & $278(7,8)$ & $29(5,7)$ & $307(7,5)$ \\
Total & $3.575(100)$ & $510(100)$ & $4.085(100)$ \\
\hline$\chi^{2} 3 G=33,43 ; p<0,0001$. & \\
\hline
\end{tabular}




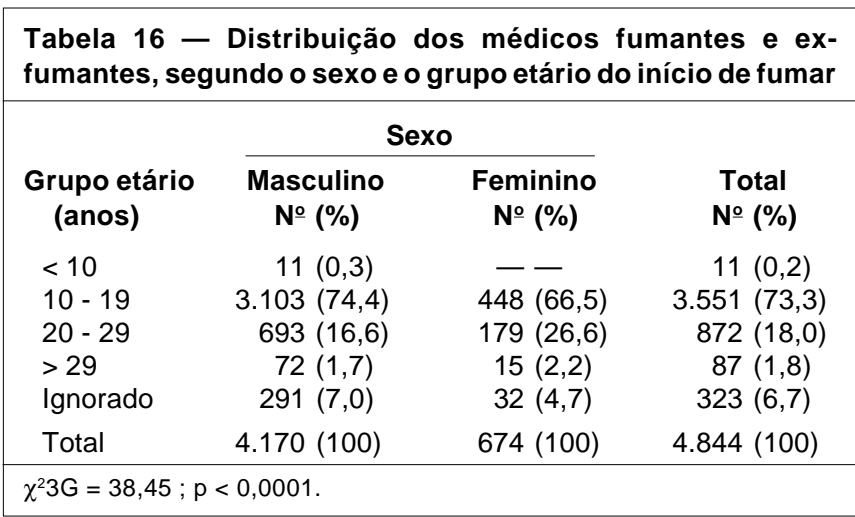

acesso mais fácil, com respostas espontâneas e voluntárias, pode-se concluir seu resultado como indicação do que ocorre na população-alvo.

Considerando-se a prevalência de fumantes regulares (759 médicos - 6,4\%) e de ex-fumantes (4.085 médicos - 34,3\%), verificou-se que $40,7 \%$ de médicos fumantes e ex-fumantes são coincidentes com os obtidos em inquéritos realizados anteriormente.

O grupo etário predominante entre os fumantes é o de 35-69 anos (89,3\%), com distribuição muito semelhante em ambos os sexos. Notou-se que no grupo etário menor de 35 anos houve predominância de médicas, quando comparado com o grupo etário maior de 69 anos, que apresenta maior freqüência do sexo masculino, diferenças estatisticamente significativas.

Com referência à especialidade médica, observou-se que aquelas com características de menor relação direta com os pacientes tiveram maior freqüência de fumantes, como genética clínica (14,3\%), admi nistração em saúde (12,5\%) e medi ci na legal $(11,1 \%)$. Outrossim, as sociedades de especialidades que possuem programas efetivos de controle do tabagismo mostraram índices menores de fumantes entre seus associados, como pneumologia/tisiologia (2,2\%), cancerologia $(3,7 \%)$, cardiologia $(4,3 \%)$ e otorrinolaringologia $(4,3 \%)$.

As maiores prevalências de fumantes foram verificadas nas U nidades da Federação do Acre, Maranhão, Mato Grosso, Mato Grosso do Sul, Pará Piauí, Rondônia e Roraima (8,2\% a 40\%), pertencentes às regiões geográficas Norte, Nordeste e Centro-Oeste, sugerindo uma situação de nãoimplantação ou ausência de efetividade dos programas de combate ao tabagismo.

Embora os médicos fumantes tenham iniciado seu vício, mais frequentemente, no grupo de 10-19 anos $(72,6 \%)$, a distribuição foi semelhante em ambos os sexos. Não se constatou nenhuma médica que tenha iniciado o fumar antes dos 10 anos de idade, não havendo, porém, diferenças significativas para ambos os sexos nos demais grupos etários.
Entre os médicos ex-fumantes, a distribuição, segundo o sexo e a idade, foi semelhante à dos fumantes regulares; as diferenças verificadas nos ex-tabagi stas foram estatisticamente significantes.

Os menores índices de ex-fumantes, segundo a especialidade médica, foram: infectologia, cirurgi a da cabeça e pescoço, medicina legal, dermatologia e administração em saúde $(20,7 \%$ a $25 \%)$, ao contrário daquelas cujas sociedades de especialidades mantêm programas de controle ao tabagismo, como pneumologia/tisiologia (36,3\%), cardiologia $(34,5 \%)$, cancerologia $(32,1 \%)$ e otorrinolaringologia $(31,6 \%)$.

I gualmente, verificaram-se menores índices de ex-fumantes nas U nidades da Federação: Roraima, Rondônia, Paraíba, Sergi pe, Alagoas, Pará, Rio Grande do Norte (20\% a 30,1\%), das regiões geográficas Norte e Nordeste. A idade em que inici ou fumar, neste grupo, foi muito semel hante aos fumantes regulares; estatisticamente, foram significantes as diferenças entre os sexos dos exfumantes.

Considerando-se os grupos de médicos fumantes e ex-fumantes, a distribuição dos grupos etários do início de fumar foi muito semel hante.

Os médicos não-fumantes têm o grupo etário de 35-69, também predominante, com distribuição muito semel hante entre os sexos. O sexo feminino apresentou maior índice no grupo etário menor de 35 anos, ao contrário do grupo etário maior de 69 anos. Neste grupo de não-fumantes, não se notam grandes diferenças nas especialidades médicas, na distribui ção das U nidades da F ederação e áreas geográficas do país.

Da análise deste estudo, conclui-se que é de grande importância levar aos médicos informações sobre as repercussões do fumar para a saúde, bem como a estruturação dos programas de controle do tabagismo nas soci edades de especialidades e nas federadas da AMB.

\begin{tabular}{|lccr|}
\hline \multicolumn{4}{|l|}{$\begin{array}{l}\text { Anexo } 1 \text { - Distribuição dos médicos participantes do } \\
\text { inquérito sobre prevalência do tabagismo, segundo as }\end{array}$} \\
regiões geográficas do país \\
\hline $\begin{array}{l}\text { Região } \\
\text { geográfica }\end{array}$ & $\begin{array}{r}\text { № de } \\
\text { respostas }\end{array}$ & $\begin{array}{c}\text { No da } \\
\text { população-alvo }\end{array}$ & $\%$ \\
Norte & 175 & 2.085 & 8,4 \\
Nordeste & 1.460 & 7.731 & 18,9 \\
Centro-Oeste & 734 & 3.855 & 19,0 \\
Sudeste & 6.352 & 27.122 & 23,4 \\
Sul & 3.188 & 10.765 & 29,6 \\
Total & 11.909 & 51.558 & 23,1 \\
\hline
\end{tabular}


Anexo 2 - Distribuição dos médicos participantes do inquérito sobre prevalência do tabagismo, segundo as federadas da $A M B$

\begin{tabular}{|lrcr|}
\hline \multicolumn{1}{c}{$\begin{array}{c}\text { Estado da } \\
\text { Federação }\end{array}$} & $\begin{array}{c}\text { No de } \\
\text { respostas }\end{array}$ & $\begin{array}{c}\text { No da } \\
\text { população-alvo }\end{array}$ & \multicolumn{1}{c|}{$\%$} \\
Acre & 5 & 92 & 5,4 \\
Alagoas & 57 & 444 & 12,8 \\
Amapá & 3 & 103 & 2,9 \\
Amazonas & 47 & 1.296 & 3,6 \\
Bahia & 423 & 1.802 & 23,5 \\
Ceará & 100 & 1.308 & 7,6 \\
Distrito Federal & 233 & 1.728 & 13,5 \\
Espírito Santo & 239 & 787 & 30,4 \\
Goiás & 228 & 988 & 23,1 \\
Maranhão & 12 & 69 & 17,4 \\
Mato Grosso & 46 & 343 & 13,4 \\
Mato Grosso do Sul & 227 & 796 & 28,5 \\
Minas Gerais & 1.187 & 5.196 & 22,8 \\
Pará & 60 & 298 & 20,1 \\
Paraíba & 150 & 849 & 17,7 \\
Paraná & 900 & 3.300 & 27,3 \\
Pernambuco & 257 & 1.238 & 20,8 \\
Piauí & 110 & 452 & 24,3 \\
Rio de Janeiro & 564 & 3.285 & 17,2 \\
Rio Grande do Norte & 206 & 1.061 & 19,4 \\
Rio Grande do Sul & 1.674 & 5.349 & 31,3 \\
Rondônia & 44 & 168 & 26,2 \\
Roraima & 5 & 67 & 7,5 \\
Santa Catarina & 614 & 2.116 & 29,0 \\
São Paulo & 4.362 & 17.854 & 24,4 \\
Sergipe & 145 & 508 & 28,5 \\
Tocantins & 11 & 61 & 18,0 \\
Total & 11.909 & 51.558 & 23,1 \\
\hline
\end{tabular}

\section{Anexo 4 - Formulário padrão utilizado no inquérito sobre prevalência do tabagismo na classe médica brasileira}

1. Nome:

2. Idade: anos

3. Sexo: $\left.\right|_{\_} \mid$Feminino $\left|\_\right|$Masculino

4. Especialidade:
5. É fumante regular:

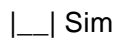
L $\mid$ Não
6. Já foi fumante e abandonou:
L Sim
| $\mid$ Não

7. Em caso de resposta afirmativa aos itens 5 e 6 , com que idade começou a fumar? anos

8. Onde exerce sua atividade médica?

$$
\text { Cidade: }
$$

Estado
Anexo 3 - Distribuição dos médicos participantes do inquérito sobre prevalência do tabagismo, segundo as especialidades

\begin{tabular}{|c|c|c|c|}
\hline $\begin{array}{l}\text { Estado da } \\
\text { Federação }\end{array}$ & $\begin{array}{l}\text { № de } \\
\text { respostas }\end{array}$ & $\begin{array}{c}\text { № da } \\
\text { população-alvo }\end{array}$ & $\%$ \\
\hline Administração em Saúde & 56 & 56 & 100,0 \\
\hline Alergologia/Imunopatologia & 51 & 386 & 13,2 \\
\hline Anestesiologia & 453 & 5.028 & 9,0 \\
\hline Cancerologia & 109 & 350 & 31,1 \\
\hline Cardiologia & 682 & 2.423 & 28,1 \\
\hline Cirurgia Cabeça e Pescoço & 28 & 206 & 13,6 \\
\hline Cirurgia Cardiovascular & 31 & 222 & 14,0 \\
\hline Cirurgia Digestiva & 77 & 454 & 17,0 \\
\hline Cirurgia Geral & 569 & 1.462 & 38,9 \\
\hline Cirurgia Neurológica & 125 & 644 & 19,4 \\
\hline Cirurgia Pediátrica & 62 & 254 & 24,4 \\
\hline Cirurgia Plástica & 201 & 1.233 & 16,3 \\
\hline Cirurgia Vascular / Angiologia & 169 & 350 & 48,3 \\
\hline Clínica Médica & 1.285 & 1.285 & 100,0 \\
\hline Dermatologia & 375 & 1.971 & 19,0 \\
\hline Endocrinologia/Metabologia & 209 & 816 & 25,6 \\
\hline Endoscopia & 38 & 617 & 6,2 \\
\hline Gastroenterologia & 344 & 460 & 74,8 \\
\hline Genética Clínica & 7 & 9 & 77,8 \\
\hline Geriatria & 81 & 208 & 38,9 \\
\hline Ginecologia / Obstetrícia & 1.642 & 4.932 & 33,3 \\
\hline Hematologia/Hemoterapia & 77 & 206 & 37,4 \\
\hline Homeopatia & 95 & 180 & 52,8 \\
\hline Infectologia & 58 & 77 & 75,3 \\
\hline Mastologia & 24 & 412 & 5,8 \\
\hline Medicina de Tráfego & 2 & 335 & 0,6 \\
\hline Medicina Desportiva & 3 & 69 & 4,3 \\
\hline Medicina do Trabalho & 133 & 647 & 20,6 \\
\hline Medicina Intensiva / UTI & 14 & 1.240 & 1,1 \\
\hline Medicina Legal & 9 & 215 & 4,2 \\
\hline Nefrologia & 136 & 548 & 24,8 \\
\hline Neurologia / Neurofisiologia & 202 & 517 & 39,1 \\
\hline Nutrologia & 5 & 61 & 8,2 \\
\hline Oftalmologia & 537 & 3.460 & 15,5 \\
\hline \multicolumn{4}{|l|}{ Ortopedia/Traumatologia/ } \\
\hline Reumatologia & 576 & 3.486 & 16,5 \\
\hline Otorrinolaringologia & 320 & 886 & 36,1 \\
\hline Patologia / Citopatologia & 130 & 741 & 17,5 \\
\hline Patologia Clínica & 125 & 683 & 18,3 \\
\hline Pediatria & 1.425 & 7.511 & 19,0 \\
\hline Pneumologia / Tisiologia & 273 & 2.067 & 13,2 \\
\hline Psiquiatria & 484 & 1.195 & 40,5 \\
\hline Radiologia & 341 & 1.981 & 17,2 \\
\hline Urologia & 219 & 1.675 & 13,1 \\
\hline Especialidade ignorada & 127 & -- & 一- \\
\hline Total & 11.909 & 51.558 & 23,1 \\
\hline
\end{tabular}

\section{AGRADE CIMENTOS}

Os autores agradecem a inestimável colaboração e o apoio técnico de Donaldo Botel ho Veneziano, Edison J esus Ventura, I ris Falconi, Maria de Fátima S.S. Nascimento, Paulo Fernandes, Valeria Lombardo, e a J osé Maria Pacheco e Souza e Maria Cristina Marcondes Malerbi, pela análise estatística dos dados e revisão do texto. 


\section{SUMMARY}

\section{Survey on prevalence of smoking among Brazil-} ian physicians

Овj Ectives. To present theresults of thestudy of the current prevalence of smoking among Brazilian physicians, according to field of specialization. The study was performed in the period from May to October 1996.

Method. Data were collected by spontaneous reply from 11,909 physicians registered in Brazilian Medical Association, in the various States of Brazil and medical specializations.

Results. The results indicated that 759 (6.4\%) are regular smokers, $11,150(93.6 \%)$ are non-smokers and that 4,085 (34.3\%) areformer smokers. The highest rates of non smoking were found among the societies of special ties that have effetive smoking control programs (pneumology, oncology, cardiology and otorhinolaryngology). The northern, northeastern and mid-western regions of the country presented the highest prevalences of smoking. The onset of smoking occurs more frequently in the 10-19 years age group (72.6\%), with similar distribution in both sexes. There were no female physicians who began smoking before 10 years of age.

Conclusion. It is very important that physicians receive information regarding smoking as well as to have the development of smoking control programs in the specialty societies and in the regional associations federated to the Brazilian Medical Association. [Rev Ass Med Brasil 1997;
43(3): 209-16.]

KEY WoRdS: Smoking. Prevalence. Brazilian Physicians.

\section{REFERÊNCIAS BIBLIOGRÁFICAS}

1. Rosemberg J , Silva ULC. Responsabilidade dos médicos no combate ao tabagismo. Monografia, Corporação Biogalênica, São Paulo, SP, 1993.

2. RosembergJ. Cartilha sobretabagismo para profissionais da saúde. Monografia, Secretaria da Saúde / CETAB, São Paulo, SP, 1995.

3. Saltz R, Gadia CANJ . Doutor, osenhor ainda fuma? Estudo do comportamento de uma população médica. J Pneumol 1981; 7: 98-101.

4. Rosemberg J. A propósito do inquérito piloto da AMB sobre o tabagismo entre os médicos. Rev Ass Med Brasil 1983; 29: 127-8.

5. Rosemberg J . Por quemédicos continuam a fumar? (E ditorial) Rev Paul Med 1988; 106: 179-82.

6. Rabelo LV. Programa Estadual de Combate ao Fumo, Pernambuco. Pesquisa sobre o hábito de fumar entre médicos. Anais da 3a Reunião Brasileira de Programas de Combate ao Fumo, Salvador, BA, 1989.

7. Campos HS, Sobrinho AP. Tabagismo entre os médicos de Sobradinho, DF, 1989. RBCTA 1991; 20: 132-9.

8. Campos HS. Tabagismoentreos médicos doBrasil.J Pneumol 1992; 18: 1-9.

9. Campos HS. Tabagismo na classe médica do Estado do Rio de J aneiro. Pulmão (RJ ) 1993; 4(2): 13-26.

10. Campos HS. Tabagismo entre pneumologistas. Resultados preliminares. Bol Pneum Sanit 1995, 1: 47-55.

11. Campos HS. Tabagismo entreos congressistas do V Congresso de Pneumologia e Tisiologia do Rio de J aneiro. Pulmão (RJ ) 1996; 5(3): 72-5.

12. Harvey L, Shubert S. AMA survey of physician and public opinion on health care-issues, 1987. American Medical Association, Chicago, 1987. 